2012-06-06

\title{
When is general wariness favored in avoiding multiple predator types?
}

\author{
Brilot, BO
}

http://hdl.handle.net/10026.1/2996

$10.1086 / 665648$

Am Nat

All content in PEARL is protected by copyright law. Author manuscripts are made available in accordance with publisher policies. Please cite only the published version using the details provided on the item record or document. In the absence of an open licence (e.g. Creative Commons), permissions for further reuse of content should be sought from the publisher or author. 


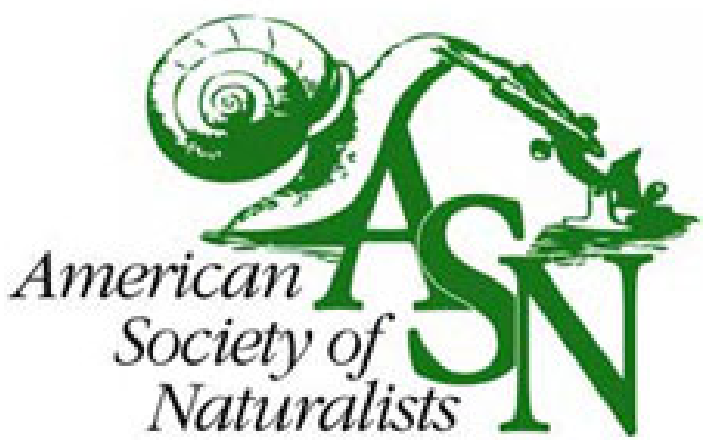

When Is General Wariness Favored in Avoiding Multiple Predator Types?

Author(s): Ben O. Brilot, Melissa Bateson, Daniel Nettle, Mark J. Whittingham, Jenny C. A.

Read, Associate Editor: Vlastimil Křivan, Editor: Ruth G. Shaw

Reviewed work(s):

Source: The American Naturalist, (-Not available-), p. 000

Published by: The University of Chicago Press for The American Society of Naturalists

Stable URL: http://www.jstor.org/stable/10.1086/665648

Accessed: 30/04/2012 05:13

Your use of the JSTOR archive indicates your acceptance of the Terms \& Conditions of Use, available at http://www.jstor.org/page/info/about/policies/terms.jsp

JSTOR is a not-for-profit service that helps scholars, researchers, and students discover, use, and build upon a wide range of content in a trusted digital archive. We use information technology and tools to increase productivity and facilitate new forms of scholarship. For more information about JSTOR, please contact support@ jstor.org. 


\title{
When Is General Wariness Favored in Avoiding Multiple Predator Types?
}

\author{
Ben O. Brilot, ${ }^{1, \star}$ Melissa Bateson, ${ }^{1}$ Daniel Nettle, ${ }^{1}$ Mark J. Whittingham, ${ }^{2}$ and Jenny C. A. Read ${ }^{3}$ \\ 1. Centre for Behavior and Evolution, Institute of Neuroscience, Henry Wellcome Building, Newcastle University, Framlington Place, \\ Newcastle upon Tyne NE2 4HH, United Kingdom; 2. School of Biology, Ridley Building, Newcastle University, Newcastle upon Tyne \\ NE1 7RU, United Kingdom; 3. Institute of Neuroscience, Henry Wellcome Building, Newcastle University, Framlington Place, \\ Newcastle upon Tyne NE2 4HH, United Kingdom
}

Submitted November 5, 2011; Accepted January 19, 2012; Electronically published April 27, 2012

Online enhancement: appendix.

\begin{abstract}
Adaptive responses to predation are generally studied assuming only one predator type exists, but most prey species are depredated by multiple types. When multiple types occur, the optimal antipredator response level may be determined solely by the probability of attack by the relevant predator: "specific responsiveness." Conversely, an increase in the probability of attack by one predator type might increase responsiveness to an alternative predator type: "general wariness." We formulate a mathematical model in which a prey animal perceives a cue providing information on the probability of two predator types being present. It can perform one of two evasive behaviors that vary in their suitability as a response to the "wrong" predator type. We show that general wariness is optimal when incorrect behavioral decisions have differential fitness costs. Counterintuitively, difficulty in discriminating between predator types does not favor general wariness. We predict that where responses to predator types are mutually exclusive (e.g., referential alarm-calling), specific responsiveness will occur; we suggest that prey generalize their defensive responses based on cue similarity due to an assumption of response utility; and we predict, with relevance to conservation, that habituation to human disturbance should generalize only to predators that elicit the same antipredator response as humans.
\end{abstract}

Keywords: multiple predators, general wariness, antipredator responses, referential alarm calls, human disturbance, predator generalization.

\section{Introduction}

Animals possess numerous adaptive mechanisms that function in avoiding predation (Lima and Dill 1990), including behavioral (Cresswell 1993; Lima and Bednekoff 1999), morphological (Harvell 1986; Hoso and Hori 2008), physiological, neurobiological (Mitra et al. 2009), and lifehistory traits (Basolo 2008; Lima 2009). For simplicity, the adaptive value of these traits is usually studied in the con-

\footnotetext{
* Corresponding author; e-mail: ben.brilot@ncl.ac.uk.
}

Am. Nat. 2012. Vol. 179, pp. 000-000. (C) 2012 by The University of Chicago. 0003-0147/2012/17906-53433\$15.00. All rights reserved. DOI: $10.1086 / 665648$ text of an environment with only a single predatory species present. However, different predatory species can coexist in the same environment and can be defined as "types" on the basis of, for example, their direction of attack (aerial vs. terrestrial), their method of hunting (sit-and-wait vs. searching), their habitat preferences (open vs. closed), the specificity of their prey preferences, changes in prey-size preference with age or sex, and so forth. Sih et al. (1998) discuss the difficulties in defining and assessing the number of predator types but conclude that the "typical prey species" probably faces attack from many types. The effects of multiple predator types on antipredator mechanisms are unlikely to be simply additive (e.g., Sih et al. 1998; Soluk 1993), hence a theoretical framework is required to derive predictions regarding the optimal level of an antipredator trait in the presence of multiple predators (e.g., Lima 1992; Matsuda et al. 1993; Blumstein et al. 2006). In this article we specifically examine the circumstances under which the optimal level of a trait designed to avoid a particular predator type is altered by changes in the probability of occurrence of an alternative predator type.

Where only one predator type exists, the optimal level of an antipredator trait is conceptually simple. If the probability of attack by that predator increases, the prey should be more likely to engage in defensive behavior directed against that predator. For instance, a foraging passerine (e.g., a house sparrow, Passer domesticus) seeing an aerial predator (e.g., a sparrowhawk, Accipiter nisus) passing at a distance could be generally extra vigilant, spending more time with its head up, scanning for an aerial threat, and be more responsive (i.e., taking flight earlier) to any approaching aerial object. This scenario is more complicated if the prey can be uncertain whether the predator is actually present; in this case, there is the potential for "false alarms" to occur: taking flight when the predator is not actually present. These false alarms are both energetically expensive and carry opportunity costs (e.g., loss of foraging time). 
Hence, the prey sets some threshold of responding that optimizes its long-term fitness (i.e., that balances the cost of false alarms against the cost of predation; Ydenberg and Dill 1986).

Where multiple predator types exist, an antipredator response to one type may vary in its utility for avoiding other types. For example, an increase in the prey's vigilance behavior could defend equally well against another type of aerial predator (e.g., a merlin, Falco columbarius) but could be as bad as doing nothing when avoiding terrestrial predators (e.g., a domestic cat, Felis catus). Hence, it is possible that the optimal level of an antipredator response to a sparrowhawk (for example) may depend on both the probability of attack by sparrowhawks and the probability of attack by other predator types. Whether this is the case is hard to intuit, and this scenario therefore requires a formal mathematical exploration.

We are particularly interested in whether, and when, the optimal strategy for an animal includes aspects of "general wariness." The terms "wariness" or "fearfulness" have previously been used to refer to the general level of response mounted by a particular individual or species to a range of potentially threatening environmental stimuli (Boissy 1995; Blumstein 2006a). We co-opt the distinct term "general wariness" from Adams et al. (2006) and use it to describe the situation where variation in the probability of attack by predator type A influences the probability that a prey animal produces an evasive response to predator type B, with all other conditions being held equal. (Below, we flesh out this initial definition with more precision.) For example, if the prey displays general wariness, then an increase in the probability of attack from a sparrowhawk may lower its threshold for flying away from cats, even though there has been no change in the probability of attack by cats. This general wariness strategy is in contrast to the situation where the prey's decision to take evasive action for predator type $B$ is unaffected by variation in the probability of attack by predator type A: "specific responsiveness." In this case, the prey's decision about whether to respond as if only a hawk is present depends solely on the probability that only a hawk is actually present. When and why would general wariness be an appropriate strategy for an animal to adopt?

\section{Modeling Framework}

We employ signal detection theory (Green and Swets 1966; Macmillan and Creelman 1991) to explore a simple model in which a foraging prey animal (e.g., a passerine) faces two predator types, has two types of signal available from the environment, and has three possible types of behavior. Each signal is actually an inadvertent cue, produced by and associated predominantly (though to a varying degree) with a particular predator (e.g., a "cat" signal and a "hawk" signal). The three behaviors are evasive action directed against each predator type ("anticat" and "antihawk"), plus the prey's default behavior in the absence of any threat (e.g., foraging). The world can be in one of four possible states (henceforth indicated by capital letters): no predators are present (NONE); one of either predator type is present (CAT, HAWK); or both types are present (BOTH; see table 1). We shall assume that the expected fitness costs of a particular behavior in a particular state are fixed. Thus, for any given situation (e.g., hawk present, cat absent), there is a clearly defined optimal behavior.

\section{Signals Cueing the Presence of a Predator}

Since the environment is assumed to be "noisy," the prey animal does not know the state of the world with certainty. We assume instead that it has access to two basic types of temporally defined information: long-term, baseline (prior) information on predation probability (we term this the "wider context") and current (posterior) information on predation probability. The first represents an assumption that the prey is storing and aggregating information (genetically or cognitively) on the general probability of predators in the environment. The second type of information is provided by moment-by-moment signals or cues from the environment. We assume that signals from the environment can change rapidly, on the same timescale as the prey must react, while the wider context changes over much longer timescales, for example, over the diurnal or seasonal cycle. Crucially, the signals available from the environment do not indicate for sure whether, for example, a hawk is present; they only convey some information about the instantaneous probability that a hawk is present. The prey has to use these probabilistic cues, together with its stored knowledge regarding the wider context, to decide what behavior is optimal for the current situation.

Formally, we represent the signal available to the prey as a two-dimensional (2D) vector, $\mathbf{s}$, defined by the magnitude of the signal relating to each cue type: $\mathbf{s}=$ $\left(s_{\text {cat }}, s_{\text {hawk }}\right)$. The signal conveys information about the presence of predators because the probability distribution from which the signal is drawn depends on the state of the world. We model this dependence very simply, assuming that $s_{\text {cat }}, s_{\text {hawk }}$ are drawn independently from Gaussian distributions with means $\bar{s}_{\text {cat }}^{\text {STATE }}$ and $\bar{s}_{\text {hawk }}^{\text {STATE }}$ (where STATE can take the values NONE, CAT, HAWK, BOTH) and unit standard deviation. Setting the standard deviation to the same value for both signal and noise distributions is a simplifying assumption, but setting it to the same value for the signal distributions for both predators involves no loss of generality, since the salient factor for these is the ratio of the mean to the standard deviation. Thus, the 
Table 1: Model parameters, notation and baseline values

\begin{tabular}{|c|c|c|}
\hline Model parameter & Notation & $\begin{array}{c}\text { Baseline } \\
\text { paramete } \\
\text { values }\end{array}$ \\
\hline \multicolumn{3}{|l|}{ State of the world: } \\
\hline STATE & Current situation or state of reality; can be NONE, CAT, HAWK or BOTH & N/A \\
\hline NONE & Situation in which no predators are present & N/A \\
\hline CAT & Situation in which only a cat-type predator is present & N/A \\
\hline HAWK & Situation in which only a hawk-type predator is present & N/A \\
\hline BOTH & Situation in which both a cat- and a hawk-type predator are present & N/A \\
\hline \multicolumn{3}{|c|}{ Environmental signals available: } \\
\hline s & Vector representing the signals currently available from the environment & N/A \\
\hline$s_{\text {cat }}$ & Strength of the signal predominantly associated with cat-type predators & N/A \\
\hline$S_{\text {hawk }}$ & Strength of the signal predominantly associated with hawk-type predators & N/A \\
\hline$\overline{\mathbf{s}}^{\mathrm{NONE}}$ & Mean signal vector when no predators are present & $(1,1)$ \\
\hline$\overline{\mathbf{s}}^{\mathrm{CAT}}$ & Mean signal vector when only a cat is present & $(3,1)$ \\
\hline$\overline{\mathbf{s}}^{\mathrm{HAWK}}$ & Mean signal vector when only a hawk is present & $(1,3)$ \\
\hline$\overline{\mathbf{s}}^{\mathrm{BOTH}}$ & Mean signal vector when both a cat and a hawk are present & $(3,3)$ \\
\hline \multicolumn{3}{|c|}{$\begin{array}{l}\text { Information regarding the state } \\
\text { of the world: }\end{array}$} \\
\hline$P($ STATE $)$ & A priori probability of the STATE situation & \\
\hline$p_{\text {cat }}$ & $\begin{array}{l}\text { Prior probability that a cat is present, assumed to be independent of } \\
\text { whether hawks are also present }\end{array}$ & .1 \\
\hline$p_{\text {hawk }}$ & $\begin{array}{l}\text { Prior probability that a hawk is present, assumed to be independent of } \\
\text { whether cats are also present }\end{array}$ & .1 \\
\hline$P(\mathrm{NONE})$ & Prior probability that no predators are present & .81 \\
\hline$P(\mathrm{HAWK})$ & Prior probability that only a hawk is present & .09 \\
\hline$P(\mathrm{CAT})$ & Prior probability that only a cat is present & .09 \\
\hline$P(\mathrm{BOTH})$ & Prior probability that both predator types are present & .01 \\
\hline$P(\mathrm{STATE} \mid \mathbf{s})$ & The posterior probability of the STATE situation, given the signal vector $\mathbf{s}$ & N/A \\
\hline$P(\mathrm{NONE} \mid \mathbf{s})$ & For example, $P(\mathrm{HAWK} \mid \mathbf{s})$ is the posterior probability that only a hawk is & \\
\hline$P($ HAWK $\mid \mathbf{s})$ & present, taking into account both the prior probability of hawks occur- & \\
\hline$P(\mathrm{CAT} \mid \mathbf{s})$ & ring and the information available from environmental cues & \\
\hline$P(\mathrm{BOTH} \mid \mathbf{s})$ & & \\
\hline \multicolumn{3}{|c|}{$\begin{array}{l}\text { Fitness payoffs for behavioral } \\
\text { decisions given the state of } \\
\text { the world (see table } 2 \text { ): }\end{array}$} \\
\hline$w($ none $\mid \mathrm{NONE})$ & Fitness payoff for continuing to forage, i.e., taking no evasive action & 0 \\
\hline$w($ none $\mid \mathrm{CAT})$ & Fitness payoff for taking no action when only a cat is present & -10 \\
\hline$w($ none $\mid \mathrm{HAWK})$ & Fitness payoff for taking no action when only a hawk is present & -10 \\
\hline$w($ none $\mid \mathrm{BOTH})$ & Fitness payoff for taking no action when both predators are present & -12 \\
\hline$w($ cat $\mid \mathrm{NONE})$ & Fitness payoff for an unnecessary anticat response & -1 \\
\hline$w($ cat $\mid \mathrm{CAT})$ & Fitness payoff for recognizing and avoiding a cat & -1 \\
\hline$w($ cat $\mid \mathrm{HAWK})$ & Fitness payoff for acting inappropriately in avoiding a hawk & -10 \\
\hline$w($ cat $\mid \mathrm{BOTH})$ & Fitness payoff for avoiding a cat but failing to avoid a hawk & -12 \\
\hline$w($ hawk $\mid \mathrm{NONE})$ & Fitness payoff for an unnecessary antihawk response & -1 \\
\hline$w(h a w k \mid \mathrm{CAT})$ & Fitness payoff for acting inappropriately in avoiding a cat & -10 \\
\hline$w(h a w k \mid$ HAWK $)$ & Fitness payoff for recognizing and avoiding a hawk & -1 \\
\hline$w($ hawk|BOTH $)$ & Fitness payoff for avoiding a hawk but failing to avoid a cat & -12 \\
\hline
\end{tabular}

probability density for the $2 \mathrm{D}$ signal s, when the current state of the world is STATE, is

$$
P(\mathbf{s} \mid \mathrm{STATE})=P\left(s_{\text {cat }} \mid \mathrm{STATE}\right) \times P\left(s_{\text {hawk }} \mid \mathrm{STATE}\right),
$$

where

$$
P\left(s_{\text {cat }} \mid \text { STATE }\right)=\frac{1}{\sqrt{2 \pi}} \exp \left[-0.5\left(s_{\text {cat }}-s_{\text {cat }}^{\mathrm{STATE}}\right)^{2}\right]
$$

and similarly for $\mathrm{s}_{\text {hawk }}$ (fig. 1 ).

Since we have chosen to keep a constant unit standard 


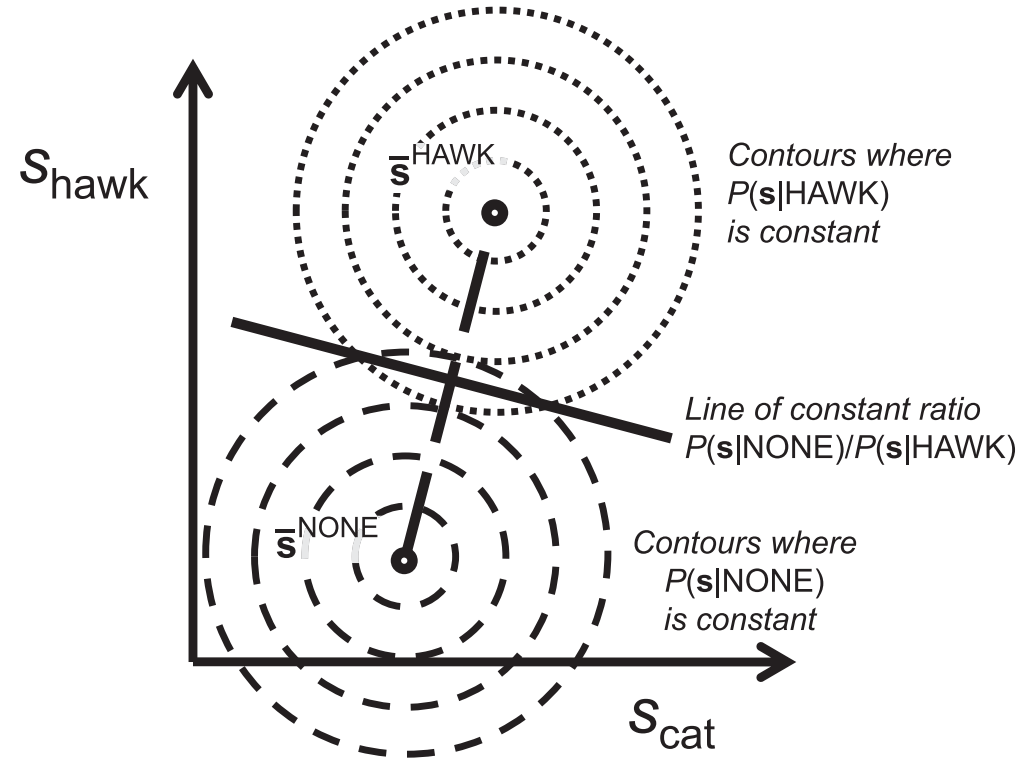

Figure 1: A sample none/hawk decision threshold when cats are irrelevant. Loci of constant posterior probability ratio $P(\mathbf{s} \mid \mathrm{NONE}) / P(\mathbf{s} \mid \mathrm{HAWK})$ are straight lines in signal space, orthogonal to the expected signal vector produced by a hawk relative to the noise signal, that is, the line joining $\overline{\mathbf{s}}^{\mathrm{NONE}}$ to $\overline{\mathbf{s}}^{\mathrm{HAWK}}$.

deviation, the clarity of the signals is set by the values chosen for their means. Since there are two signal components and four states, we need to specify eight values of $\bar{s}_{\text {component }}^{\text {STATE }}$. These correspond to the positions of four points on the two-dimensional space representing the signal (fig. 2). Since the mean signal in the NONE state defines a baseline, the behavior of the model is defined by the remaining 6 independent values (plus the payoff matrix, see below).

\section{Independent versus Ambiguous Signals}

We have labeled the two components of the signal $s_{\text {cat }}$ and $s_{\text {hawk }}$, assuming that each is indicative predominantly of one particular predator type. The degree to which this is true is set by the values chosen for the means, $\overline{\boldsymbol{s}}^{\text {STATE }}$, in each world-state. If each signal component is caused by only one predator type, then the mean value of $s_{\text {cat }}$ depends only on whether or not a cat is present, and the mean value of $s_{\text {hawk }}$ depends only on whether or not a hawk is present. Graphically, in this case the lines joining $\overline{\mathbf{s}}^{\mathrm{NONE}}$ to $\overline{\boldsymbol{s}}^{\text {HAWK }}$ and $\overline{\boldsymbol{s}}^{\mathrm{CAT}}$ will be parallel to the $s_{\text {hawk }}$ and $s_{\text {cat }}$ axes, respectively (as in fig. 2). When this is not the case, at least one of the predators produces a signal that, on average, comprises both signal components. We refer to this as cue ambiguity and consider specific examples in "Results" (fig. 3B).

In nonmathematical language, each predator can cause, in general, two types of signals indicating its presence, for example, a shadow and a rustling noise. However, we can vary how strongly the signals are associated with one or the other predator. For example, in the "separate signals" condition we can set the model such that the hawk causes a strong "shadow" signal and no "rustle" signal (and vice versa for the cat; fig. 2). At the other extreme, the cues from each predator are exactly the same (maximally ambiguous) and differentiating them on the basis of the received signal is impossible. Between these extremes each signal is in general associated more strongly with one or the other predator; for example, a hawk, may normally cause a strong shadow signal and only a weak rustle signal.

\section{A Priori Probabilities}

As well as constantly updated information provided by these signals, we assume that the prey also has separate prior probability estimates for each predator in the model (the "wider context"). In all the simulations presented in this article, these priors are independent: that is, the a priori probability that both predators are present is simply the product of the a priori probability that either individually is present. Thus, if we write $p_{\text {cat }} p_{\text {hawk }}$ for the a priori probability of each predator type, then 


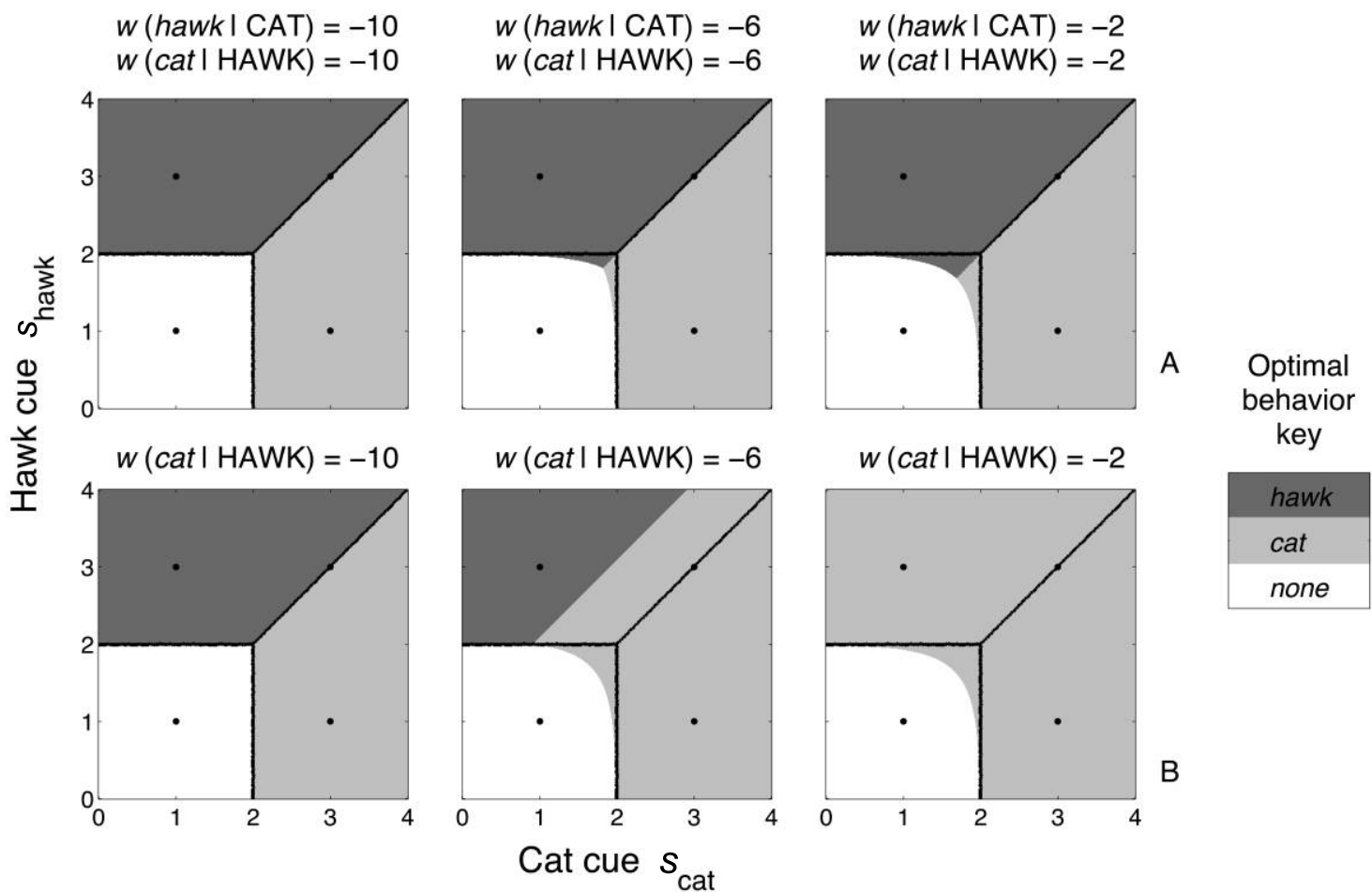

Figure 2: A, Reducing the inappropriateness of active responses to the incorrect predator favors general wariness as the optimal defensive strategy. The horizontal axis shows the strength of the "cat" signal, $s_{\text {cat }}$, and the vertical axis shows the strength of the "hawk" signal, $s_{\text {hawk }}$, and these constitute the vector $\mathbf{s}$. The shading represents the optimal behavior of the prey for each $\mathbf{s}$ (see the adjacent key): none $=$ no antipredator response; $c a t=$ anticat behavior; hawk $=$ antihawk behavior. For comparison, the decision thresholds from the left-hand column are overlaid on subsequent panels (as black lines). Black dots indicate $\overline{\mathbf{s}}^{\mathrm{NONE}}$ (bottom left), $\overline{\boldsymbol{s}}^{\mathrm{HAWK}}$ (top left), $\overline{\mathbf{s}}^{\mathrm{CAT}}$ (bottom right), and $\overline{\mathbf{s}}^{\text {вОтн }}($ top right $)$. The model parameter values are as per table 1 except for those indicated above each panel; in particular, $w($ none $\mid$ CAT) and $w($ none $\mid \mathrm{HAWK})$ are held constant at -10 throughout. $B$, Reducing the inappropriateness of one of the active responses while holding the other constant favors general wariness but only in the general antipredator response. The model parameter values are as per table 1 except for those indicated above each panel.

$$
\begin{aligned}
P(\mathrm{CAT}) & =p_{\text {cat }} \times\left(1-p_{\text {hawk }}\right), \\
P(\mathrm{HAWK}) & =p_{\text {hawk }} \times\left(1-p_{\text {cat }}\right), \\
P(\mathrm{BOTH}) & =p_{\text {cat }} \times p_{\text {hawk }}, \\
P(\mathrm{NONE}) & =\left(1-p_{\text {cat }}\right) \times\left(1-p_{\text {hawk }}\right),
\end{aligned}
$$

where $P(\mathrm{CAT})$ and $P(\mathrm{HAWK})$ are the exclusive prior probabilities of the cat and hawk, respectively, occurring; $P(\mathrm{BOTH})$ is the prior probability that they both occur; and $P(\mathrm{NONE})$ is the prior probability that neither is present.

The prey combines information provided by the two signals with its own stored priors to estimate the probability that each predator type is present. According to Bayes's theorem, the posterior probability that the world is in a particular state (e.g., only cat present), given a particular signal $\mathbf{s}$, is proportional to

$$
P(\text { STATE } \mid \mathbf{s})=\frac{P(\text { STATE }) \times P(\mathbf{s} \mid \text { STATE })}{P(\mathbf{s})},
$$

where

$$
P(\mathbf{s})=\sum_{\text {STATE }} P(\text { STATE }) P(\mathbf{s} \mid \text { STATE }) .
$$

Term $P($ STATE $)$ is the a priori probability of a particular state. As outlined above, $P(\mathbf{s} \mid \mathrm{STATE})$ is the probability of receiving a signal $\mathbf{s}$ when the world is in that state (eq. $[1])$, and $P(\mathbf{s})$ is the a priori probability of obtaining the particular signal vector $\mathbf{s}$.

\section{Payoff Matrix}

The optimal behavior in any given situation depends not only on these probabilities but on the expected costs associated with each behavior. Given a particular signal vec- 


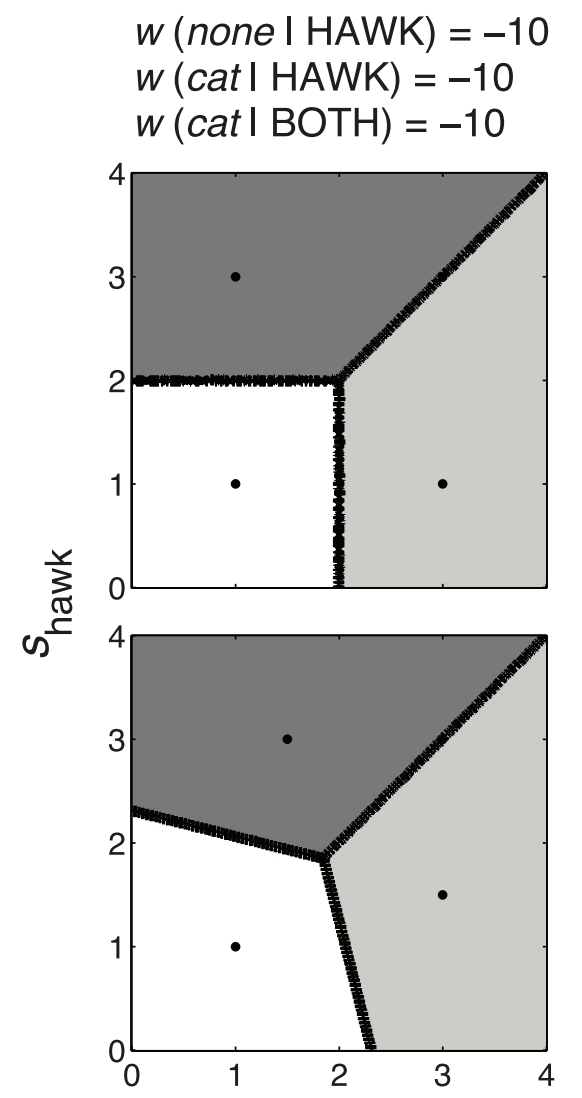

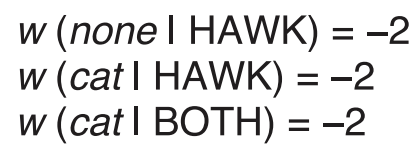
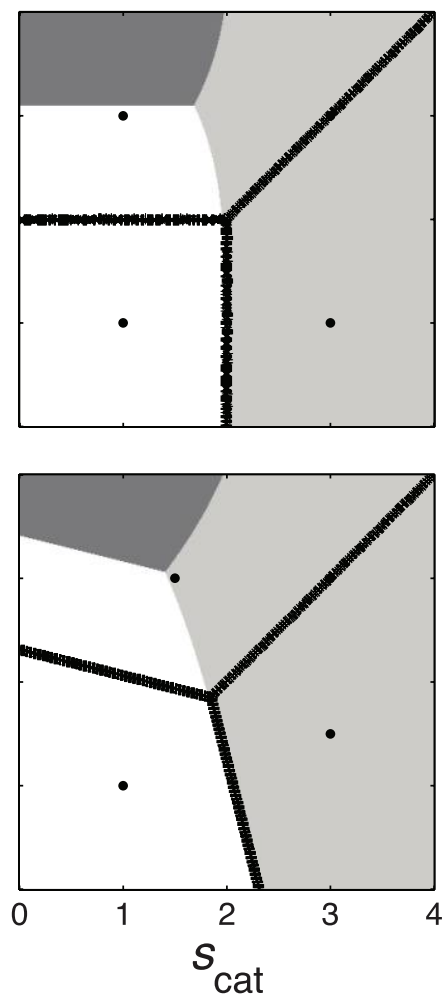

$w($ none $\mathrm{IHAWK})=0$

$w($ cat I HAWK $)=-1$

$w($ cat $\mid \mathrm{BOTH})=-1$

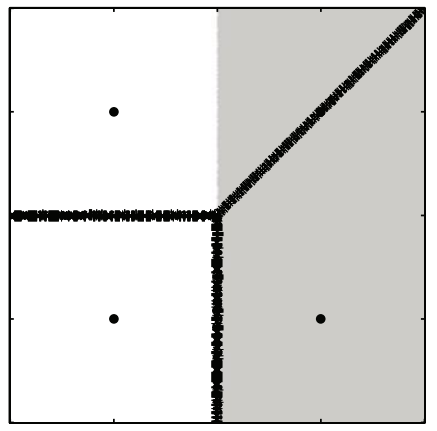

A

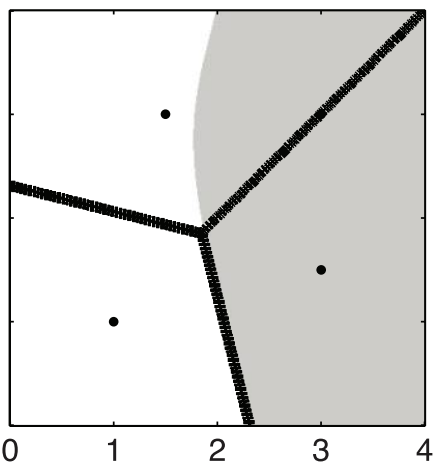

$\mathrm{B}$

Figure 3: $A$, Decreasing the danger posed by one of the predators (the hawk) can lead to general wariness but only in its response to the more dangerous predator (the cat) and only when the less dangerous predator is relatively innocuous. The prey does not respond to cues of the less dangerous predator once it is completely innocuous. $B$, Decreasing the danger posed by one of the predators (the hawk) causes a very small favoring of general wariness when predator cues are ambiguous $\left(\overline{\mathbf{s}}^{\mathrm{CAT}}=(3,1.5), \overline{\mathbf{s}}^{\mathrm{HAWK}}=(1.5,3)\right)$. However, ambiguous cues from an entirely innocuous "predator" can inhibit the active response to a dangerous predator. The model parameter values are as per table 1 except for those indicated above each column; those defining cue ambiguity are as described above; and $w($ none $\mid \mathrm{BOTH})=-10$, $w($ hawk|BOTH $)=-10$.

tor, $\mathbf{s}=\left(s_{\text {cat }}, s_{\text {hawk }}\right)$, the prey can make three potential responses (actions are denoted in lower case): continue foraging, none; perform behavior cat, a suitable response to a cat (e.g., fly into a tall tree); or perform behavior hawk, a suitable response to a hawk (e.g., fly into dense cover). As per the standard signal detection theory model, each of these behaviors carries some payoff dependent on their level of suitability as a response given the real state of the world. We write $w$ (action|STATE) for the utility of behavior action in situation STATE (see tables 1,2).

Different choices of utility value for $w$ describe different situations, for example, the relative danger of each predator and the protection offered by each behavior. In order to make the model suitable for the predation situation we have described, we require at least the following relationships:

$$
w(\text { cat } \mid \mathrm{CAT}) \geq w(\text { none } \mid \mathrm{CAT})
$$

(behavior described as anticat must be better than doing nothing when a cat is present),

$$
w(\text { cat } \mid \mathrm{CAT}) \geq w(\text { cat } \mid \mathrm{HAWK})
$$

(behavior described as anticat must not be less useful against cats than hawks), with equivalent relationships holding for the antipredator behavior: hawk. All the simulations presented in this article satisfy these relationships. We shall discuss further conditions below. 
Table 2: Payoff matrix for the behavior adopted in response to the perceived signal, given the actual stimulus present

\begin{tabular}{|c|c|c|c|c|}
\hline & \multicolumn{4}{|c|}{ Stimulus present (STATE) } \\
\hline & $\begin{array}{c}\text { No predator present } \\
\text { (NONE) }\end{array}$ & $\begin{array}{l}\text { Only cat present } \\
\text { (CAT) }\end{array}$ & $\begin{array}{l}\text { Only hawk present } \\
\text { (HAWK) }\end{array}$ & $\begin{array}{l}\text { Both predators } \\
\text { present } \\
\text { (BOTH) }\end{array}$ \\
\hline \multicolumn{5}{|c|}{ Action taken (action): } \\
\hline Behavior none & $\begin{array}{l}\text { Correct rejection } \\
\quad w(\text { none } \mid \mathrm{NONE})\end{array}$ & Miss $w($ none $\mid \mathrm{CAT})$ & Miss $w($ none $\mid \mathrm{HAWK})$ & Miss $w($ none $\mid \mathrm{BOTH})$ \\
\hline Behavior cat & $\begin{array}{l}\text { False alarm } \\
\qquad w(c a t \mid \mathrm{NONE})\end{array}$ & $\begin{array}{l}\text { Hit (i.e., avoid predator) } \\
\quad w(\text { cat } \mid \mathrm{CAT})\end{array}$ & $\begin{array}{l}\text { Inappropriate active re- } \\
\text { sponse } w(\text { cat } \mid \mathrm{HAWK})\end{array}$ & $\begin{array}{l}\text { Inappropriate active } \\
\text { response } \\
w(\text { cat } \mid \mathrm{HAWK})\end{array}$ \\
\hline Behavior hawk & $\begin{array}{l}\text { False alarm } \\
\qquad w(h a w k \mid \mathrm{NONE})\end{array}$ & $\begin{array}{l}\text { Inappropriate active re- } \\
\text { sponse } w(\text { hawk } \mid \mathrm{CAT})\end{array}$ & $\begin{array}{l}\text { Hit (i.e., avoid predator) } \\
\qquad w(\text { hawk } \mid \text { HAWK })\end{array}$ & $\begin{array}{l}\text { Inappropriate active } \\
\text { response } \\
w(\text { hawk } \mid \mathrm{CAT})\end{array}$ \\
\hline
\end{tabular}

\section{Expected Payoffs}

We can now define the expected fitness payoffs, $W($ action $\mid \mathbf{s})$, if the prey performs behavior action in response to the particular signal vector $\mathbf{s}$. This is simply the fitness payoff for that behavior given that the world is in a particular state, weighted by the probability that the world is in that state, averaged over the four possible states:

$$
\begin{aligned}
W(\text { action } \mid \mathbf{s})= & \sum_{\text {TTATE }} P(\text { STATE } \mid \mathbf{s}) \\
& \times w(\text { action } \mid \text { STATE }) .
\end{aligned}
$$

The probability of each state is derived from the available signals and the predator prior probabilities, as specified in equation (4). Thus, we can expand equation (6) into

$$
\begin{aligned}
W(\text { action } \mid \mathbf{s})= & \frac{1}{P(\mathbf{s})} \sum_{\text {STATE }} P(\text { STATE }) \\
& \times P(\mathbf{s} \mid \text { STATE }) \times w(\text { action } \mid \text { STATE }) .
\end{aligned}
$$

\section{Calculating the Optimal Response}

We define the optimal response as that which yields the maximum expected utility (Green and Swets 1966), that is, the action that maximizes $W($ action $\mid \mathbf{s})$ for the given signal vector $\mathbf{s}$. All payoffs are inversely proportional to the a priori probability of the signal, $P(\mathbf{s})$, but since this is the same for all actions, it does not affect which response is optimal and thus has no effect on our results. We solve numerically to find the optimal response for each signal vector over the range from $(0,0)$ to the upper bound (defined by the mean signal vector when either one or both predators are present, plus one standard deviation of the noise). The results are represented graphically in decision graphs such as figure 2 . These graphs show the optimal behavioral response for a given signal vector $\mathbf{s}$, shown over the range of values $\mathbf{s}$ can take.

\section{Location of the Decision Thresholds}

In reality, the prey's antipredator behavior may vary in a continuous fashion (e.g., scanning frequency) but in our simplified model, the prey switches between discrete behavioral choices. Thus the prey's overall behavioral strategy is defined by the decision thresholds, the key signal values at which it switches from one behavior to another. These decision thresholds are marked in the left-hand panel in figure $2 A$ with heavy black lines separating the shaded regions.

\section{Defining General Wariness and Specific Responsiveness}

Given our model premise, we can now begin to define the terms "general wariness" and "specific responsiveness" more precisely. We say that the prey is responding specifically to hawks if the decision threshold between its default behavior none and antihawk action hawk is unaffected by the existence of cats. For a prey animal demonstrating specific responsiveness to hawks, the none/hawk threshold is independent of the prior probability of cats, $p_{\text {cat. }}$ That is, the decision threshold for hawk behavior for any value of $p_{\text {cat }}$ is the same as for $p_{\text {cat }}=0$.

The decision thresholds correspond to an exact balance between the expected fitness costs of the two behaviors. The decision threshold between none and hawk actions, for example, is given by the values of the signal vectors $s$ which satisfy

$$
W(\text { none } \mid \mathbf{s})=W(\text { hawk } \mid \mathbf{s}) .
$$

From equation (7) we see that this requires 


$$
\begin{gathered}
\frac{1}{P(\mathbf{s})} \sum_{\text {STATE }} P(\text { STATE }) P(\mathbf{s} \mid \text { STATE }) \\
\times[w(\text { none } \mid \text { STATE })-w(\text { ha } w k \mid \text { STATE })]=0 .
\end{gathered}
$$

Note that the value of $\mathrm{P}(\mathbf{s})$, being the same for all possible actions at a given signal value, cancels out. Then expanding out the sum over possible states, we have

$$
\begin{gathered}
P(\mathrm{NONE} \mid \mathbf{s})[w(\text { none } \mid \mathrm{NONE})-w(\text { ha } w k \mid \mathrm{NONE})] \\
+P(\mathrm{HAWK} \mid \mathbf{s})[w(\text { none } \mid \mathrm{HAWK})-w(\text { ha } w k \mid \mathrm{HAWK})] \\
+P(\mathrm{CAT} \mid \mathbf{s})[w(\text { none } \mid \mathrm{CAT})-w(\text { ha } w k \mid \mathrm{CAT})] \\
+P(\mathrm{BOTH} \mid \mathbf{s})[w(\text { none } \mid \mathrm{BOTH})-w(\text { haw } w \mid \mathrm{BOTH})]=0 .
\end{gathered}
$$

If cats never occur, then both $P(\mathrm{CAT} \mid \mathbf{s})$ and $P(\mathrm{BOTH} \mid \mathbf{s})$ are zero. The none/hawk decision boundary is therefore simply the set of signal values $\mathbf{s}$ satisfying

$$
\begin{gathered}
\frac{P(\mathrm{HAWK} \mid \mathbf{s})}{P(\mathrm{NONE} \mid \mathbf{s})} \\
=\frac{[w(\text { none } \mid \mathrm{NONE})-w(\text { haw } k \mid \mathrm{NONE})]}{[w(\text { hawk } \mid \mathrm{HAWK})-w(\text { none } \mid \mathrm{HAWK})]} .
\end{gathered}
$$

Equation (10) relates to the situation when cats do not exist $\left(p_{\text {cat }}=0\right)$. In this case, it is obvious that the decision to switch from the default behavior none to evasive behavior hawk should depend only on the relative probabilities that the coast is clear or that a hawk is present, given the available information, that is, on $P(\mathrm{HAWK} \mid \mathbf{s}) / P(\mathrm{NONE} \mid \mathbf{s})$. If an animal's none/hawk decision threshold continues to depend only on the ratio $P(\mathrm{HAWK} \mid \mathbf{s}) / P(\mathrm{NONE} \mid \mathbf{s})$ even when cats do exist $\left(p_{\text {cat }}>\right.$ 0 ), we define the animal as responding specifically to the threat of hawks. Note that hawk is defined to be the appropriate behavior when only a hawk is present. It is not appropriate behavior when both predators are present. Indeed, in our default set of parameters given in table 1, the utility of behavior hawk when both predators are present is less than its utility when only a cat is present $(w($ hawk $\mid \mathrm{HAWK})=-1, w($ hawk $\mid \mathrm{CAT})=-10, w$ hawk $\mid$ $\mathrm{BOTH})=-12$ ). This is why the relevant probability ratio for the none/hawk transition is $P(H A W K \mid \mathbf{s}) / P(\mathrm{NONE} \mid \mathbf{s})$, not $[P(\mathrm{HAWK} \mid \mathbf{s})+P(\mathrm{BOTH} \mid \mathbf{s})] / P(\mathrm{NONE} \mid \mathbf{s})$, even when $P(\mathrm{BOTH} \mid \mathbf{s})$ is nonzero.

If the prey's decision to switch from its default behavior to targeted antihawk behavior depends not only on the ratio $P(\mathrm{HAWK} \mid \mathbf{s}) / P(\mathrm{NONE} \mid \mathbf{s})$ but also on $P(\mathrm{CAT} \mid \mathbf{s})$ and/ or $P(\mathrm{BOTH} \mid \mathbf{s})$, then we say the prey is displaying general wariness. In this case, the location of the prey's none/hawk decision threshold will depend not only on the prior prob- ability of hawks occurring but also on the prior probability of cats occurring.

\section{Conditions Required for Specific Responsiveness}

We can now examine the circumstances under which the prey would respond specifically to hawks, apparently acting as if cats did not exist when deciding when to switch from behavior none to behavior hawk. From equation (9), we can see that for this to occur when $p_{\text {cat }}>0$, two conditions must be met:

$$
\begin{aligned}
w(\text { none } \mid \mathrm{CAT}) & =w(\text { haw } k \mid \mathrm{CAT}), \\
w(\text { none } \mid \mathrm{BOTH}) & =w(\text { haw } k \mid \mathrm{BOTH}) .
\end{aligned}
$$

That is, specific responsiveness to hawks occurs when antihawk defensive action offers no protection against cats (eq. [11a]) and is as bad as taking no action if both predator types are present (eq. [11b]). In that case, the likelihood of cats is irrelevant when considering when to switch between none and hawk. Therefore, substituting equations (11) into equation (9) results in the same decision threshold as before, equation (10), even when $p_{\text {cat }}>0$. If this is not the case-for example, if hawk offers some protection against cats-then the animal should take the prior probability of cats occurring into account. The decision threshold will then not be given by equation (10).

By the same reasoning, the prey should respond specifically to cats when

$$
\begin{aligned}
w(\text { none } \mid \mathrm{HAWK}) & =w(\text { cat } \mid \mathrm{HAWK}), \\
w(\text { none } \mid \mathrm{BOTH}) & =w(\text { cat } \mid \mathrm{BOTH}) .
\end{aligned}
$$

If these conditions do not hold then the prey will display general wariness in its cat response.

\section{Graphical Signature of Specific Responsiveness}

Conveniently, in our model specific responsiveness has a clear signature on our decision graphs, which allows us to tell simply by inspecting a decision graph whether equation (10) holds or not (examining the effect of changing prior probabilities would be equivalent but more complicated; see fig. A1, available online).

From equation (4), the ratio of probabilities on the lefthand side of equation (10) can be rewritten as

$$
\frac{P(\text { HAWK } \mid \mathbf{s})}{P(\text { NONE } \mid \mathbf{s})}=\frac{P(\text { HAWK }) P(\mathbf{s} \mid \text { HAWK })}{P(\text { NONE }) P(\mathbf{s} \mid \text { NONE })} .
$$

Therefore, when cats do not exist, the none/hawk decision boundary is given by the signal values satisfying 


$$
\begin{gathered}
\frac{P(\mathbf{s} \mid \mathrm{HAWK})}{P(\mathbf{s} \mid \mathrm{NONE})} \\
=\frac{P(\mathrm{NONE})[w(\text { none } \mid \mathrm{NONE})-w(\text { haw } k \mid \mathrm{NONE})]}{P(\mathrm{HAWK})[w(\text { haw } k \mid \mathrm{HAWK})-w(\text { none } \mid \mathrm{HAWK})]} .
\end{gathered}
$$

Substituting from equation (3), confirms that this ratio is independent of $p_{\text {cat }}$ :

$$
\begin{gathered}
\frac{P(\mathbf{s} \mid \mathrm{HAWK})}{P(\mathbf{s} \mid \mathrm{NONE})} \\
=\frac{\left(1-p_{\text {hawk }}\right)[w(\text { none } \mid \mathrm{NONE})-w(\text { haw } k \mid \mathrm{NONE})]}{p_{\text {hawk }}[w(\text { hawk } \mid \mathrm{HAWK})-w(\text { none } \mid \mathrm{HAWK})]} .
\end{gathered}
$$

In our model, the distributions $P(\mathbf{s} \mid \mathrm{STATE})$ are Gaussian. If we substitute for $P(\mathbf{s} \mid \mathrm{STATE})$ from equation (1) and equation (2) into equation (13) and solve, we find that the none/hawk decision threshold is given by a straight line. We can see this graphically in figure 1. Loci of constant $P(\mathbf{s} \mid$ STATE $)$ are given by circles centered on $\bar{s}^{\text {STATE }}$. The ratio of $P(\mathbf{s} \mid \mathrm{HAWK})$ to $P(\mathbf{s} \mid \mathrm{NONE})$ is constant along the line indicated in figure 1.

Thus, in our model, when the animal responds specifically to hawks, the none/hawk decision threshold is a straight line in signal space. This straight line must be orthogonal to the line joining the mean signal when only a hawk is present to the mean signal when no predator is present. This neat result immediately enables us to tell simply by looking at a graph of the decision thresholds in signal space to see whether the prey responds specifically to hawk threats. It does so if, and only if, the none/hawk decision threshold is a straight line orthogonal to the imaginary line joining the mean signal values for the NONE and HAWK states. Similarly, the prey responds specifically to cat threats if, and only if, the none/cat decision threshold is a straight line (again, orthogonal to the line joining the mean signal for only a cat present to the mean signal produced when no predator is present). If both these thresholds are straight lines the prey responds specifically to each threat. If either or both are curved, the prey displays some form of general wariness. As we will see in "Results," specific responsiveness and general wariness relate to each particular predator type since the optimal strategy for the two need not be identical; the prey may show specific responsiveness in its decision threshold for antihawk actions, but general wariness in the decision threshold for anticat action.

\section{Results}

Since our definitions of general wariness and specific responsiveness are key to this article, we reiterate them here. Specific responsiveness occurs when the prey animal's threshold for taking antipredator behavior directed against predator $\mathrm{A}$ is independent of the prior probability of predator B, that is, is the same as if predator B did not exist. Equivalently, the decision to switch from the default behavior to anti-A behavior depends only on the ratio $P(\mathbf{s} \mid \mathrm{A}) / P(\mathbf{s} \mid \mathrm{NONE})$, not on $P(\mathbf{s} \mid \mathrm{B})$ or $P(\mathbf{s} \mid \mathrm{BOTH})$. General wariness, by contrast, is a strategy whereby the decision on whether to undertake antipredator behavior against predator A is partly dependent on the prior probability that predator $\mathrm{B}$ is present.

We can now examine the parameter spaces where specific responsiveness and/or general wariness are optimal. The default values in table 1 satisfy equations (11a)-(11d), and therefore, given this parameter set, specific responsiveness to both predators should be the optimal strategy. First we examine the effect on antipredator behavior of violating equations (11a), (11c) and (11b), (11d) separately, and subsequently, due to assumptions of biological realism, we will allow changes in the parameter set to violate all four simultaneously.

\section{Varying the Costs of Inappropriate Responses}

What if a behavior designed to avoid a cat is also partially (or wholly) successful in avoiding hawks? Formally, in the model we simultaneously increase the utility of each inappropriate action relative to the utility of a miss $(w($ hawk $\mid \mathrm{CAT})>w($ none $\mid \mathrm{CAT}), \quad w($ cat $\mid \mathrm{HAWK})>$ $w($ none $\mid \mathrm{HAWK}))$, thus violating equations (11a) and (11c). As the responses to predators become increasingly general (inappropriate actions become less costly), then general wariness comes to be favored as revealed in figure $2 A$.

We can also vary the costs of inappropriate active responses asymmetrically, for example, holding the hawk response as entirely inappropriate $(w($ hawk $\mid \mathrm{CAT})=$ $w($ none $\mid \mathrm{CAT}))$ while increasing the generality of the cat response $(w($ cat $\mid \mathrm{HAWK})>w($ none $\mid \mathrm{HAWK}))$, thus violating equation (11c) while continuing to satisfy the other conditions, equations (11a), (11b), (11d). As one active response becomes more appropriate for dealing with both predators then general wariness is increasingly favored (fig. $2 B$ ) but only in relation to the predator for which the best response is a general antipredator behavior (here, the cat). An increase in signal strength related to the hawk cue causes a decrease in the anticat decision threshold because this behavior would also partially protect against a hawk attack. Conversely, altering the signal strength relating to the cat does not change the antihawk decision threshold since this behavior would not protect against an increased chance of attack by a cat: the antihawk response is specific. As one response becomes more general to the point of being effective for all predators, then it becomes the sole 
active response, displays general wariness and has a symmetrical distribution (since both predators are equally dangerous and have equal prior probabilities; final panel in fig. $2 B)$.

\section{Varying the Costs of Responding in the Face of Multiple Predators}

Next we examine the effect of violating equations (11b), (11d) by reducing the utility $w($ none $\mid \mathrm{BOTH})$ below the values of $w($ cat $\mid \mathrm{BOTH})$ and $w($ hawk $\mid \mathrm{BOTH})$. That is, we ask whether general wariness remains optimal if, when faced with multiple predators, doing something is better than doing nothing (e.g., while holding all other payoffs constant we can increase the cost of doing nothing when two predators attack). Graphical representation of the results is not particularly illuminating (though see fig. A2, available online, included for completeness) since, though general wariness is optimal, the impact of violating equations (11b), (11d) is barely perceptible at print resolutions, particularly so when compared to the effect of violating equations (11a), (11c). In other words, the most important factor in determining general wariness is whether doing the wrong thing still has some benefit as compared to doing nothing.

\section{Varying the Ambiguity of Predator Cues}

Having examined the effect of our derived conditions (eqq. [11a]-[11d]) on the optimal defensive strategy, we move on to examine the impact of changes in predator cue ambiguity. Intuitively, before we began analyzing the model, we predicted that if predators were increasingly difficult to tell apart then they would be treated as a single predator type, resulting in general wariness. However, it is clear from the "Methods" section that this is not the case: the equations defining the optimality of specific responsiveness or general wariness are independent of any cue ambiguity. Even with ambiguous cues, so long as the responses to each predator type are still entirely specific (i.e., giving an antihawk response when a cat is present is as bad as doing nothing), then specific responsiveness is still optimal (fig. A3, available online, shows that the decision thresholds are orthogonal to the line joining the mean "noise" signal to each predator's signal vector). Even though it is increasingly difficult to discriminate between the predators, it is optimal for the prey to assume that the most likely predator type is present and respond appropriately, ignoring the alternative predator type.

\section{Simple and Realistic Sets of Utility Values}

Having examined the mathematical conditions necessary for specific responsiveness, for the remainder of our analysis we will simplify the parameter values in order to make the analysis more straightforward while respecting realistic biological assumptions. In a real biological system it is unlikely that all 12 utilities $w$ (action|STATE) described by the model would vary independently. For example, if the prey uses a particular defense (e.g., cat) when both predators are present, it should be able to evade the "appropriate" predator but may be eaten by the "inappropriate" predator. Its payoff will therefore be given by the utility of this response to the incorrect predator. Thus, from now on we assume that

$$
\begin{aligned}
w(\text { cat } \mid \mathrm{BOTH}) & =w(\text { cat } \mid \mathrm{HAWK}), \\
w(\text { hawk } \mid \mathrm{BOTH}) & =w(\text { hawk } \mid \mathrm{CAT}) .
\end{aligned}
$$

Furthermore, we assume that if the prey fails to respond when under attack by both predators, it suffers the cost associated with failing to respond to the more dangerous predator:

$$
\begin{gathered}
w(\text { none } \mid \mathrm{BOTH}) \\
=\min [w(\text { none } \mid \mathrm{CAT}), w(\text { none } \mid \mathrm{HAWK})] .
\end{gathered}
$$

These assumptions remove three degrees of freedom by making the three $w$ (action $\mid \mathrm{BOTH})$ values dependent on four of the other $w$ values.

Under these assumptions, the two conditions for specific responsiveness to each predator (eqq. [11a]-[11d]) each collapse to a single condition. When the three conditions specified in equations (14) hold, then the prey will respond specifically to hawk threats if and only if

$$
\begin{gathered}
w(\text { none } \mid \mathrm{CAT})=w(\text { haw } k \mid \mathrm{CAT}) \\
=w(\text { haw } k \mid \mathrm{BOTH}) \leq w(\text { none } \mid \mathrm{HAWK}) .
\end{gathered}
$$

Similarly it will respond specifically to cat threats if and only if

$$
\begin{gathered}
w(\text { none } \mid \mathrm{HAWK})=w(\text { cat } \mid \mathrm{HAWK}) \\
=w(\text { cat } \mid \mathrm{BOTH}) \leq w(\text { none } \mid \mathrm{CAT}) .
\end{gathered}
$$

Note that we only see specific responses to the less dangerous predator (specific responses are given to both predators if they are equally dangerous). For example, if the cat is the more dangerous predator, in the sense that $w($ none $\mid \mathrm{CAT})<w($ none $\mid \mathrm{HAWK})$, equation (15b) will not be satisfied and we will not see specific responses to cat threats. We examine this in more detail in the next section. 


\section{Varying the Threat Posed by Each Predator Type}

Finally, we relax the assumption of equally dangerous predators and allow for one predator that is less dangerous (i.e., the cost of misses (w(none|HAWK)) and inappropriate actions ( $w($ cat $\mid \mathrm{HAWK}), w($ cat $\mid \mathrm{BOTH}))$ are reduced relative to the second predator). As we make these changes, we continue to satisfy the biologically plausible conditions of equation (14); for example, we hold $w($ cat $\mid \mathrm{HAWK})$ and $w(c a t \mid \mathrm{BOTH})$ equal to each other as both are reduced. First, we examine the situation where there is no ambiguity between the cues from each predator. As the hawk becomes less dangerous, unsurprisingly, the prey chooses to respond less to this predator (fig. 3A). Counterintuitively, general wariness emerges as the hawk becomes nearly innocuous. When the threat from the relatively innocuous predator is low (i.e., the cue shows a low-to-medium probability of this predator), the prey responds with the alternative action because this carries only a low cost of being inappropriate but guards against the dangerous predator (the cat). As the cue becomes clearer for the relatively innocuous hawk, the prey begins to favor the more appropriate antihawk response. Importantly, general wariness is optimal only in relation to the more dangerous cat; the prey still shows specific responsiveness in regard to the less dangerous hawk. Once the hawk becomes completely innocuous $(w($ action $\mid \mathrm{HAWK})=w($ action $\mid \mathrm{NONE}) ;$ last panel of figure $3 A$ ) then the prey no longer shows any response to it, nor do cues of its presence alter the prey's response to the still dangerous cat.

It might seem obvious that when one predator has become completely innocuous, the system is effectively a single-predator system. However, when predator cues are ambiguous, this "obvious" result fails to hold. When cues are ambiguous, varying the danger posed by each predator has a qualitatively different effect: general wariness is marginally favored, but an innocuous second species (i.e., a nonpredator) can inhibit responding to the dangerous predator. We examine this in figure $3 B$. Here, the cues are ambiguous: the presence of the hawk causes a small increase in the mean cat signal as well as a large increase in the mean hawk signal. When both predators are equally dangerous (the first panel in fig. $3 B$ ), the prey shows specific responsiveness to both threats, with decision thresholds straight and orthogonal to the lines joining the mean signals. As one predator (the hawk) becomes decreasingly dangerous, there is a small deviation from linearity in the decision threshold for the still-dangerous predator (the cat). Significantly though, even when the hawk is completely innocuous (the final panel in fig. $3 B$ ), cues of its presence can still alter the probability of responding to the cat. This demonstrates general wariness but in a reverse sense: cues associated with an innocuous species inhibit (rather than augment) an active response to the dangerous predator. Therefore, when considering the evolution of general wariness/specific responsiveness, we must take account of not just predators but also innocuous species in the environment that might resemble predators.

\section{Discussion}

We are interested in whether or not prey integrate information on the probability of attack from multiple predator types in behavioral strategies we term "general wariness" and "specific responsiveness." The crux of these strategies is whether or not the probability of responding to predator type $\mathrm{A}$ is dependent on the prior probability of attack from an alternative predator type B. The prey shows general wariness if the probability of responding to predator type A varies with changes in the prior probability of attack from predator type $B$; the prey shows specific responsiveness if the former is independent of the latter.

We have derived two conditions that must hold for specific responsiveness to be the optimal strategy: a behavior designed to avoid predator type A must be wholly ineffective against predator type B (as bad as doing nothing), and a behavior designed to avoid one predator type must be wholly ineffective when both predators are present (employing a defensive behavior against predator type A is as bad as doing nothing if both predator types attack simultaneously). Numerical simulation reveals that the former condition has the greatest impact on determining the degree of general wariness exhibited in the system. In other words, the more that antipredator behaviors become general (suitable for avoiding all predator types), the more the probability of attack from one predator type will influence the level of response to an alternative predator type.

Our overarching finding is that general wariness will be adaptive whenever prey are faced with some uncertainty about the presence of multiple predator types (a signal detection problem), when prey can make mistakes in responding to each type, and when those mistakes have differing fitness costs (i.e., an inappropriate response is much cheaper than doing nothing). This explains why the degree of cue ambiguity is irrelevant to the optimal strategy (figs. A3, A4). Even when very uncertain about which predator is present, it is best for the prey to respond with an action appropriate to whichever is more likely to be present, ignoring changes in the probability of the less likely predator. Additionally, the differential utility of mistakes explains how general wariness and specific responsiveness can coexist as behavioral strategies when predator types are differentially dangerous or antipredator responses are differentially general (one specific and one general). Interestingly, in the former case an increase in the prior 
probability of a less dangerous predator type can increase the level of responding to the more dangerous predator type, but cues from an innocuous species (rather than a predator) can inhibit responding to the real predator (so long as cues are ambiguous; see table 3 for a full results summary).

\section{Previous Theoretical Models}

The findings from our model tally with, and extend beyond, previous models examining the impact of multiple predators (e.g., Lima 1992; Matsuda et al. 1993). For instance, Lima (1992) focused on the degree of vigilance that animals should direct towards two separate predator types and examined whether predictions varied dependent on whether vigilance was nonspecific (it allows simultaneous detection of both threats) or specific (vigilance directed at one threat precludes the ability to detect the alternative). His model demonstrated that increases in predation risk from the presence of a second predator type generally cause an increase in nonspecific vigilance levels relative to those in the presence of only one predator type. This accords with our finding that when an antipredator response is effective against multiple predators (i.e., "inappropriate" actions are nearly or as effective as appropriate actions), general wariness is favored. Additionally, Lima (1992) found that under nonspecific vigilance, even a relatively innocuous predator type could have a large effect on increasing vigilance levels. We concur that nearly innocuous predators can increase responding to other (more dangerous) predators, but interestingly, we found this effect can hold even when cues are unambiguous and incorrect responses are entirely inappropriate (fig. $3 A$ ).

A complementary theoretical approach-the multipredator hypothesis-has examined the impact on prey behavior of shifting selection pressures in multipredator systems, that is, the loss or gain of some or all of a prey species' predators (e.g., in island populations; Blumstein et al. 2006). It highlights the interplay of selection and genetic effects in maintaining antipredator behavioral complexes (Blumstein 2006b) and may explain why antipredator responses are mounted to rarely encountered predators or innocuous taxa (e.g., Webb et al. 2009). Our approach differs in that we assume that selection is constant and there are no constraints limiting the attainability of the optimal response to multiple predators (see Grafen 2007). There is value in both approaches, but we focus here on the evolution of general wariness given our model predictions based on behavioral optimality. Below we discuss how our predictions regarding the occurrence of general wariness can be helpful in understanding current empirical work.

\section{Relevant Empirical Results}

In contrast to the limited amount of theoretical research regarding multiple predators, there has been a slew of empirical research addressing their behavioral and eco-

Table 3: Key model findings in the context of the utility of two antipredator responses for avoiding two predator types, A and B, and the characteristics of those predator types

\begin{tabular}{|c|c|c|c|}
\hline Utility of antipredator responses & $\begin{array}{l}\text { Predator-cue } \\
\text { ambiguity }\end{array}$ & $\begin{array}{l}\text { Relative danger posed } \\
\text { by each predator }\end{array}$ & Optimal behavior \\
\hline Specific to predator A or predator B & $\begin{array}{l}\text { No ambiguity, some } \\
\text { ambiguity }\end{array}$ & Equal & Specific responsiveness \\
\hline $\begin{array}{l}\text { Response to each predator has some } \\
\text { utility in avoiding the alternative } \\
\text { predator }\end{array}$ & $\begin{array}{l}\text { No ambiguity, some } \\
\text { ambiguity }\end{array}$ & Equal & General wariness \\
\hline $\begin{array}{l}\text { One general response that is effective } \\
\text { for avoiding predators } \mathrm{A} \text { and } \mathrm{B}\end{array}$ & $\begin{array}{l}\text { No ambiguity, some } \\
\text { ambiguity }\end{array}$ & Equal & General wariness \\
\hline $\begin{array}{l}\text { Response to predator A has utility in } \\
\text { avoiding predator B; response to } \\
\text { predator B is specific }\end{array}$ & $\begin{array}{l}\text { No ambiguity, some } \\
\text { ambiguity }\end{array}$ & Equal & $\begin{array}{l}\text { General wariness in response to pred- } \\
\text { ator A, specific responsiveness to } \\
\text { predator B }\end{array}$ \\
\hline Specific to predator A or predator B & $\begin{array}{l}\text { No ambiguity, some } \\
\text { ambiguity }\end{array}$ & $\begin{array}{l}\text { Predator A more } \\
\text { dangerous }\end{array}$ & $\begin{array}{l}\text { General wariness in response to pred- } \\
\text { ator A, specific responsiveness to } \\
\text { predator B }\end{array}$ \\
\hline Specific to predator A or predator B & No ambiguity & $\begin{array}{l}\text { Predator A dangerous, } \\
\text { "predator" B } \\
\text { innocuous }\end{array}$ & $\begin{array}{l}\text { Specific responsiveness (no response } \\
\text { to "predator" B given) }\end{array}$ \\
\hline Specific to predator A or predator B & Some ambiguity & $\begin{array}{l}\text { Predator A dangerous, } \\
\text { "predator" B } \\
\text { innocuous }\end{array}$ & $\begin{array}{l}\text { General wariness in the sense that } \\
\text { cues from "predator" B inhibit re- } \\
\text { sponding to predator A }\end{array}$ \\
\hline
\end{tabular}


logical impacts. However, there are few studies (that we know of) that have altered the perception of the probability of attack by one predator type and examined the prey species' response to an alternative predator type, thereby providing a direct test of general wariness as defined above. If our model is correct, then studies involving prey species with differential responses to different predator types should show specific responsiveness (with general predator-response species showing general wariness).

The appropriate empirical studies, though limited in number, tentatively uphold the above theoretical predictions. Cheney and Seyfarth (1988) tested the generalization of habituation of vervet monkeys (Cercopithecus aethiops) to conspecific predator-specific alarm calls (those elicited by either a leopard [Panthera pardus] or martial eagle [Polemaetus bellicosus] which in turn prompt mutually exclusive responses). Repeated exposure to an alarm call leads to reduced defensive responding but this habituation effect only generalized to novel alarm calls that relate to the same class of predators (leopard/eagle) as the habituating call. Seyfarth and Cheney (1990) then used the same paradigm to examine the response of vervets to heterospecific (superb starlings [Lamprotornis superbus]) terrestrial/aerial alarm calls. They found that habituation to starling aerial alarm calls did not generalize to vervet terrestrial alarm calls but habituation to starling "terrestrial" alarm calls does generalize to vervet aerial alarm calls. They proposed that generalization of habituation does occur in the second case since the starling "terrestrial" alarm call is given in response to a wide range of predators and nonpredators, both aerial and terrestrial.

Hinde (1970) examined the mobbing response of chaffinches (Fringilla coelebs) to more direct predator cues: a stuffed mount of an owl or dog. He showed that the response could be enhanced by preexposure to the alternate stuffed predator (e.g., dog followed by owl). Interestingly, presentation of the owl had a greater impact on the subsequent response to the dog than the reverse presentation and was more effective in generating the mobbing response when presented in isolation, suggesting it is the more dangerous predator. Our discrete model makes an analogous prediction for this continuously varying response: that an increase in the prior probability of the more dangerous predator has a greater impact-increasing active responding across a wider range of cue stimulithan the less dangerous predator (fig. A5, available online).

Finally, Adams et al. (2006) also conducted a study looking for general wariness (though they used their own definition, their experiment equally tests our narrower definition). They found that prior exposure to raptor vocalizations could alter vigilance behavior in crimson rosellas (Platycercus elegans). However, this altered vigilance did not translate into changes in the distance at which experimental subjects took flight when approached by a human. Our model predicts a lack of general wariness in this instance due to expected differences in the utility of escape responses (terrestrial vs. aerial predator approach).

More work is clearly required to demonstrate that the model predictions hold across various multipredator systems. As the studies above show, general wariness can manifest and be measured in two ways: sensitization to predation cues from one predator type can be generalized to predation cues from a different predator type; or similarly, habituation to predation cues can be generalized between predator types. Below we outline fields of research where we believe such tests might be fruitfully conducted and where the model framework may be most usefully applied to understanding empirical systems. Amongst other things, the model supports hypotheses relating to the evolution of functionally referential signaling systems; makes predictions regarding the effects of anthropogenic disturbance; and has broader implications for the existence of generalization of antipredator behavior.

\section{Functionally Referential Alarm Calls}

Functionally referential alarm calls are specific calls given in response to differing predator types; call receivers respond to such calls with the appropriate antipredator response. The classic case of such behavior comes from vervet monkeys (as outlined above) that produce different alarm calls to leopards, martial eagles, and pythons (Python sebae; Seyfarth et al. 1980a, 1980b). Playback studies in this system confirmed that individuals respond to the alarm calls appropriately for the predatory stimulus that initiated the call (Seyfarth et al. 1980a).

It has been suggested that functionally referential alarm calls have evolved in particular species because of the incompatibility of differing responses to different classes of predators (Macedonia and Evans 1993). That is, if the utility of the correct response to different predator types does not overlap, then selection should favor additional information being provided in alarm calls indicating the correct response choice. Habitat may play a key role in shaping the general utility of antipredator responses (Macedonia and Evans 1993), in determining both suitable places of refuge and places/direction/imminence of attack (Evans 1997). Macedonia and Evans (1993) highlight the case of ring-tailed lemurs (Lemur catta) and black-andwhite ruffed lemurs (Varecia variegata variegata) which occupy differing habitats (open semiarid vs. closed canopy rainforest), are terrestrial to different degrees (partially vs. largely arboreal) and differ in the specificity (match between predator identity and alarm call given) of their aerial and terrestrial alarm calls (very specific vs. low specificity). 
Correspondingly, signal receivers in ring-tailed lemurs show a behavioral distinction when played conspecific aerial/terrestrial alarm calls whereas ruffed lemurs do not (Macedonia and Evans 1993).

The parallels between this prediction for the evolution of functionally referential alarm calling and the model predictions presented here are striking. The existence of mutually exclusive antipredator responses to different predator types might drive the evolution of such alarm calls, in tandem causing selection for specific responsiveness as a behavioral strategy (Cheney and Seyfarth 1988; though see Evans 1997) with, potentially, each type of response requiring a different physiological mechanism for its proper implementation (Mateo 2010). Referential alarm calling systems therefore probably provide the best empirical systems for testing a prediction of the existence of specific responsiveness versus general wariness (Evans 1997 provides an excellent framework for how to conduct such tests). Indeed, a more complex prediction can arise in such systems; for example, aerial predator alarm calls are given to other raptors and nonraptors that display cue similarities to the main aerial predator in vervet monkeys (fig. 1 in Seyfarth et al. 1980a). We predict that sensitization and/or habituation to cues from one predatory threat (e.g., a martial eagle) should generalize to cues from another threat that elicits the same alarm call response (e.g., another raptor species) but not to a predatory threat that elicits a different alarm call (e.g., a leopard).

\section{Cue Ambiguity and Generalization}

There are few direct tests of the prediction of sensitization/ habituation generalizing based on response utility, but there is a great deal of evidence examining whether animals generalize a predatory response (learnt or innate) from known predatory cues to cues from other, often related species (Griffin et al. 2000; Kelley and Magurran 2003; Blumstein et al. 2009). For example, fathead minnows (Pimephales promelas) when conditioned to respond to a particular predator generalize this response to cues from novel fish species (Ferrari et al. 2007). They do so in a graded fashion related to the taxonomic relationship between the conditioned and novel species (and by proxy the likely discriminability of chemical cues), not to the level of actual predation threat posed.

This principle seems contrary to our model prediction that ambiguity between predator cues is unimportant in determining general wariness: prey seem to be generalizing to ambiguous predator cues based on perceived cue similarity (Ghirlanda and Enquist 2003), regardless of the response utility. However, we argue that they may give the same (though often attenuated) response to a novel, ambiguous "predator" cue because they are expecting this response to have some utility. This is a reasonable first assumption given that predators presenting similar cues are likely to be taxonomically related, might be expected to attack in a similar fashion and therefore require the same (or very similar) response. Hence, generalization of antipredator responses may arise because, at the simplest level, ambiguous cues tend to derive from predators requiring similar defensive responses. Selection might therefore favor an initial response rule-of-thumb whereby generalization occurs on the basis of cue similarity. Learning can occur postencounter (particularly in social species) when further cues are available to confirm the accuracy and suitability of the antipredator response (Kelley and Magurran 2003).

\section{Generalization and Conservation}

Generalization of habituation carries two concerns for conservationists: reintroduction to the wild of humanreared/tended captive populations may be compromised by the "tameness" induced and their consequent lack of the response to natural predators (van Heezik et al. 1999); habituation to significant human disturbance in natural environments might lead to a reduced antipredator response in wild populations (Coleman et al. 2008; Tuomainen and Candolin 2011). The model presented confirms that both concerns are theoretically justified: habituation to humans may well be generalized, but crucially this generalization should be of most concern where a prey species has only a single, general defensive response. Those that have exclusive responses should only be generalizing their habituation to predators that require the same evasive response as humans would (terrestrial mammalian predators being the likeliest).

A secondary question arises as to whether the prey species concerned perceives cues from humans and predators as similar or otherwise. For example, human disturbance by fast-approaching or larger objects (e.g., motorized vehicles) should be more likely to initiate flight in prey animals since these are predator cues of great importance for prey (Frid and Dill 2002). However, there is some evidence that habituation to human disturbance does not necessarily lead to a lack of discrimination of predators vs. nonpredators (Coleman et al. 2008) suggesting that maybe humans and predators might be easily discriminable. We suggest that the modality of the predator cues is of paramount importance in such studies though. Visual cues (e.g., approaching humans/predator mounts) probably present sufficiently ambiguous cues to represent a genuine signal detection problem (e.g., St. Clair et al. 2010; Griffin et al. 2001). However, predator and human olfactory cues are unlikely to share sufficient ambiguities to represent even a basic signal detection problem (i.e., there 
is no overlap of the "signal" distribution). Auditory cues likely span the range from highly ambiguous (e.g., sounds of terrestrial locomotion) to completely discriminable (e.g., species-specific calls, roars; note that completely discriminable is not equivalent to "separate signals" in oul model since by completely discriminable we mean no overlap of signal distribution). Hence, empirical studies in this field must be careful to assess which modality they arc concerned with since generalization of habituation/sensitization in one modality need not necessarily correspond to generalization in another.

\section{Conclusion}

We have shown that general wariness is favored in re- $\overrightarrow{ }$ sponding to multiple predators when mistakes in discrimination are made, and those mistakes carry differential costs. We argue that this principle can explain a diverse set of empirical results deriving from the study of multipredator systems. Given the generality of the model, $\mathrm{i}-$ can also apply to related fields, such as the study of the behavioral response of animals to human disturbance. $\mathrm{Wc}_{\mathrm{c}}$ hope that the framework presented here will aid researchers trying to understand the diversity in levels of general wariness across the animal kingdom.

\section{Acknowledgments}

We thank A. Higginson, J. Lazarus, and A. Radford for fruitful discussions and advice. We are immensely gratefur to D. Blumstein and an anonymous reviewer for their reviews. This work was supported by a grant awarded to M.B. from the UK's Biotechnology and Biological Sciences Research Council (BB/E012000/1) and by the Royal Society (University Research Fellowship UF041260 tc $\rightarrow$ J.C.A.R.). multipredator hypothesis: yellow-bellied marmots respond fearfully to the sight of novel and extinct predators. Animal Behaviour 78:873-878.

Boissy, A. 1995. Fear and fearfulness in animals. Quarterly Review of Biology 70:165-191.

Cheney, D. L., and R. M. Seyfarth. 1988. Assessment of meaning and detection of unreliable signals by vervet monkeys. Animal Behaviour 36:477-486.

$\rightarrow$ Coleman, A., D. Richardson, R. Schechter, and D. T. Blumstein. 2008. Does habituation to humans influence predator discrimination in Gunther's dik-diks (Madoqua guentheri)? Biology Letters 4:250252.

$\rightarrow$ Cresswell, W. 1993. Escape responses by redshanks, Tringa totanus, on attack by avian predators. Animal Behaviour 46:609-611.

Evans, C. S. 1997. Referential signals. Perspectives in Ethology 12: 99-143.

$\rightarrow$ Ferrari, M. C. O., A. Gonzalo, F. Messier, and D. P. Chivers. 2007. Generalization of learned predator recognition: an experimental test and framework for future studies. Proceedings of the Royal Society B: Biological Sciences 274:1853-1859.

Frid, A., and L. Dill. 2002. Human-caused disturbance stimuli as a form of predation risk. Conservation Ecology 6:11.

$\rightarrow$ Ghirlanda, S., and M. Enquist. 2003. A century of generalization. Animal Behaviour 66:15-36.

Grafen, A. 2007. The formal Darwinism project: a mid-term report. Journal of Evolutionary Biology 20:1243-1254.

Green, D. M., and J. A. Swets. 1966. Signal detection theory and psychophysics. Wiley, New York.

$\rightarrow$ Griffin, A. S., D. T. Blumstein, and C. S. Evans. 2000. Training captive-bred or translocated animals to avoid predators. Conservation Biology 14:1317-1326.

$\rightarrow$ Griffin, A. S., C. S. Evans, and D. T. Blumstein. 2001. Learning specificity in acquired predator recognition. Animal Behaviour 62: 577-589.

Harvell, C. D. 1986. The ecology and evolution of inducible defenses in a marine bryozoan: cues, costs, and consequences. American Naturalist 128:810-823.

Hinde, R. A. 1970. Behavioural habituation. Pages 3-40 in G. Horn and R. A. Hinde, eds. Short-term changes in neural activity and behaviour. Cambridge University Press, Cambridge.

$\rightarrow$ Hoso, M., and M. Hori. 2008. Divergent shell shape as an antipredator adaptation in tropical land snails. American Naturalist 172: 726-732.

$\rightarrow$ Kelley, J. L., and A. E. Magurran. 2003. Learned predator recognition and antipredator responses in fishes. Fish and Fisheries 4:216-226.

\section{Literature Cited}

$\rightarrow$ Adams, J. L., K. W. Camelio, M. J. Orique, and D. T. Blumstein. 2006. Does information of predators influence general wariness? Behavioral Ecology and Sociobiology 60:742-747.

$\rightarrow$ Basolo, A. L. 2008. Evolution of pleiotropic alleles for maturation and size as a consequence of predation. Biology Letters 4:200-203.

$\rightarrow$ Blumstein, D. T. 2006a. Developing an evolutionary ecology of fear. $\rightarrow$ how life history and natural history traits affect disturbance tolerance in birds. Animal Behaviour 71:389-399.

$\rightarrow-2006 b$. The multipredator hypothesis and the evolutionar; $\rightarrow$ persistence of antipredator behavior. Ethology 112:209-217.

$\rightarrow$ Blumstein, D. T., A. Bitton, and J. DaVeiga. 2006. How does the presence of predators influence the persistence of antipredato $\rightarrow$ Mac behavior? Journal of Theoretical Biology 239:460-468.

$\rightarrow$ Blumstein, D. T., E. Ferando, and T. Stankowich. 2009. A test of the
Lima, S. L. 1992. Life in a multi-predator environment: some considerations for antipredatory vigilance. Annales Zoologici Fennici 29:217-226.

$\rightarrow-2009$. Predators and the breeding bird: behavioral and reproductive flexibility under the risk of predation. Biological Reviews 84:485-513.

Lima, S. L., and P. A. Bednekoff. 1999. Back to the basics of antipredatory vigilance: can nonvigilant animals detect attack? Animal Behaviour 58:537-543.

Lima, S. L., and L. M. Dill. 1990. Behavioral decisions made under the risk of predation: a review and prospectus. Canadian Journal of Zoology 68:619-640.

Macedonia, J. M., and C. S. Evans. 1993. Variation among mammalian alarm call systems and the problem of meaning in animal signals. Ethology 93:177-197. 


\section{The American Naturalist}

Macmillan, N. A., and C. D. Creelman. 1991. Detection theory: a user's guide. Cambridge University Press, Cambridge.

$\rightarrow$ Mateo, J. M. 2010. Alarm calls elicit predator-specific physiologica $\rightarrow$ St. responses. Biology Letters 6:623-625.

$\rightarrow$ Matsuda, H., P. A. Abrams, and H. Hori. 1993. The effect of adaptive antipredator behavior on exploitative competition and mutualism between predators. Oikos 68:549-559.

$\rightarrow$ Mitra, R., R. Adamec, and R. Sapolsky. 2009. Resilience against predator stress and dendritic morphology of amygdala neurons. Behavioural Brain Research 205:535-543.

$\rightarrow$ Seyfarth, R. M., D. L. Cheney, and P. Marler. 1980a. Monkey response to three different alarm calls: evidence of predator classification and semantic communication. Science 210:801-803.

$\rightarrow-1980 \mathrm{~b}$. Vervet monkey alarm calls: semantic communication in a free-ranging primate. Animal Behaviour 28:1070-1094.

$\rightarrow$ Seyfarth, R. M., and D. Cheney. 1990. The assessment by vervet monkeys of their own and another species' alarm calls. Anima' Behaviour 40:754-764.

$\rightarrow$ Sih, A., G. Englund, and D. Wooster. 1998. Emergent impacts of multiple predators on prey. Trends in Ecology \& Evolution 13: 350-355.

$\rightarrow$ Soluk, D. A. 1993. Multiple predator effects: predicting combined functional response of stream fish and invertebrate predators. Ecology 74:219-225.

St. Clair, J. J. H., G. E. Garcia-Pena, R. W. Woods, and T. Szekely. 2010. Presence of mammalian predators decreases tolerance to human disturbance in a breeding shorebird. Behavioral Ecology 21:1285-1292.

$\rightarrow$ Tuomainen, U., and U. Candolin. 2011. Behavioural responses to human-induced environmental change. Biological Reviews 86: 640-657.

$\rightarrow$ van Heezik, Y., P. J. Seddon, and R. F. Maloney. 1999. Helping reintroduced houbara bustards avoid predation: effective antipredator training and the predictive value of pre-release behaviour. Animal Conservation 2:155-163.

$\rightarrow$ Webb, J. K., W. G. Du, D. A. Pike, and R. Shine. 2009. Chemical cues from both dangerous and nondangerous snakes elicit antipredator behaviours from a nocturnal lizard. Animal Behaviour 77:1471-1478.

Ydenberg, R. C., and L. M. Dill. 1986. The economics of fleeing from predators. Advances in the Study of Behavior. 16:229-249.

Associate Editor: Vlastimil Křivan Editor: Ruth G. Shaw

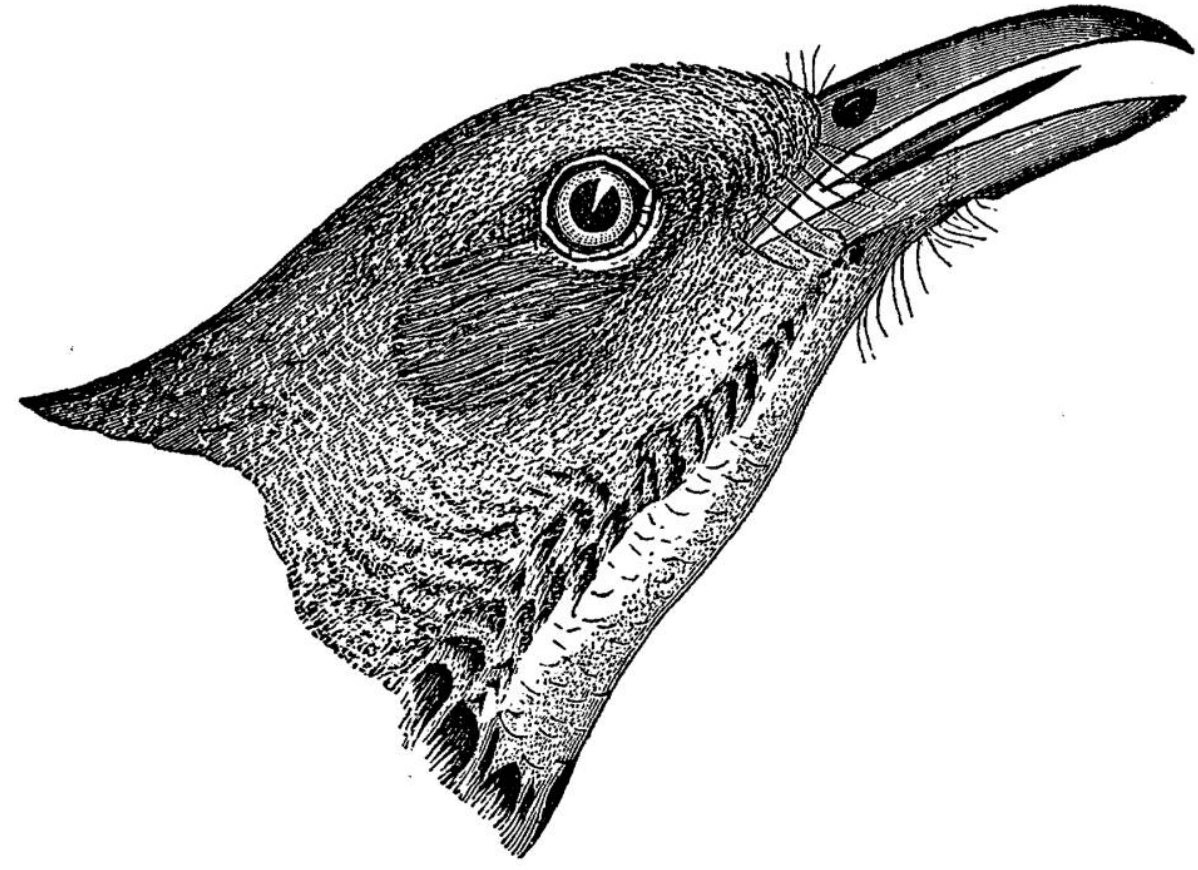

\footnotetext{
"Almost everyone knows the Brown Thrush, or Thrasher (Harporhynchus rufus) of the Eastern United States—an abundant and familiar inhabitant of shrubbery and a spirited songster, with some talent for mimicry. It belongs to the mocking-thrush group (Miminae) all of which are famous for their vocal powers; the cat-bird, and the princely mocking-bird itself, are near relatives." From "Some United States Birds, New to Science, and Other Things Ornithological” by Dr. Elliott Coues (American Naturalist, 1873, 7:321-331).
} 